\title{
Uncertainty and Sensitivity Analyses of a Two-Parameter Impedance Prediction Model
}

\author{
M. G. Jones, ${ }^{*}$ T. L. Parrott ${ }^{\dagger}$ and W. R. Watson ${ }^{\ddagger}$ \\ NASA Langley Research Center, Hampton, VA 23681-2199, USA
}

\begin{abstract}
$\mathbf{T}$ HIS paper presents comparisons of predicted impedance uncertainty limits derived from Monte-Carlotype simulations with a Two-Parameter (TP) impedance prediction model and measured impedance uncertainty limits based on multiple tests acquired in NASA Langley test rigs. These predicted and measured impedance uncertainty limits are used to evaluate the effects of simultaneous randomization of each input parameter for the impedance prediction and measurement processes. A sensitivity analysis is then used to further evaluate the TP prediction model by varying its input parameters on an individual basis. The variation imposed on the input parameters is based on measurements conducted with multiple tests in the NASA Langley normal incidence and grazing incidence impedance tubes; thus, the input parameters are assigned uncertainties commensurate with those of the measured data. These same measured data are used with the NASA Langley impedance measurement (eduction) processes to determine the corresponding measured impedance uncertainty limits, such that the predicted and measured impedance uncertainty limits $(95 \%$ confidence intervals) can be compared. The measured reactance $95 \%$ confidence intervals encompass the corresponding predicted reactance confidence intervals over the frequency range of interest. The same is true for the confidence intervals of the measured and predicted resistance at near-resonance frequencies, but the predicted resistance confidence intervals are lower than the measured resistance confidence intervals (no overlap) at frequencies away from resonance. A sensitivity analysis indicates the discharge coefficient uncertainty is the major contributor to uncertainty in the predicted impedances for the perforate-over-honeycomb liner used in this study. This insight regarding the relative importance of each input parameter will be used to guide the design of experiments with test rigs currently being brought on-line at NASA Langley.
\end{abstract}

\section{Nomenclature}

a implicit empirical constant

$A, B \quad$ linear and nonlinear parameters, respectively, $\mathrm{kg} /\left(\mathrm{m}^{2} \cdot \mathrm{s}\right)$ and $\mathrm{kg} / \mathrm{m}^{3}$

$c, \rho \quad$ ambient sound speed and density, respectively, $\mathrm{m} / \mathrm{s}$ and $\mathrm{kg} / \mathrm{m}^{3}$

$C_{D} \quad$ discharge coefficient

$d, h, t \quad$ hole diameter, cell (cavity) depth and facesheet thickness, respectively, $\mathrm{m}$

$f, \omega \quad$ frequency and angular frequency, respectively, $\mathrm{Hz}$ and $\mathrm{rad} / \mathrm{s}$

$i \quad=\sqrt{-1}$, unit imaginary number

$k \quad$ free space wavenumber, $\mathrm{m}^{-1}$

$k_{i}, k_{e} \quad$ empirical constants for hole entrance/exit end effects

$M_{\text {ave }} \quad$ uniform (average) flow Mach number

NLF nonlinearity factor

$P_{S}, T_{S} \quad$ static pressure and temperature, respectively, $\mathrm{Pa}$ and kelvins

$R, R_{f}, R_{g f} \quad$ acoustic, DC and grazing flow resistances, respectively, $\mathrm{kg} /\left(\mathrm{m}^{2} \cdot s\right)$

SPL $\quad$ sound pressure level, dB

$V_{i n c}, V_{r m s} \quad$ incident flow velocity and RMS acoustic particle velocity, respectively, $\mathrm{m} / \mathrm{s}$

\footnotetext{
* Senior Research Scientist, Research \& Technology Directorate, Structural Acoustics Branch, Mail Stop 463; Michael.G.Jones@NASA.GOV. Associate Fellow of AIAA.

$\dagger$ Senior Research Scientist, Research \& Technology Directorate, Structural Acoustics Branch, Mail Stop 463; Tony.L.Parrott@NASA.GOV.

¥Senior Research Scientist, Research \& Technology Directorate, Computational Aerosciences Branch, Mail Stop 128; Willie.R.Watson@NASA.GOV. Associate Fellow of AIAA.
} 


$\begin{array}{ll}\delta^{*} & \text { boundary layer displacement thickness, } \mathrm{m} \\ \Delta p & \text { pressure drop, Pa } \\ \varepsilon d & \text { hole length end correction in TP model } \\ \sigma & \text { open area ratio } \\ \theta, \chi & \text { normalized acoustic resistance and reactance, respectively } \\ \zeta & =\theta+i \chi, \text { normalized normal incidence impedance }\end{array}$

Note: An $e^{i \omega t}$ time convention is used throughout this paper, and all impedances are normalized by $\rho c$.

\section{Introduction}

$\mathrm{F}$ OR over four decades, perforate-over-honeycomb acoustic liners have served as a mechanically robust and acoustically effective means of suppressing fan tone noise propagating through fan inlet and exhaust ducts in commercial aircraft turbofan engines. Their mechanical robustness stems from the honeycomb "sandwich" construction, whereas their locally-reacting, resonant acoustic response provides acceptable tonal suppression for a range of liner impedances. In light of the inherent fluid dynamical issues associated with modeling the effects of high acoustic levels and mean flow velocities for perforate-over-honeycomb liners, this acoustic effectiveness has proven to be invaluable. However, the transition to high-bypass ratio turbofan engines has caused a decrease in the ratio of the length of the liner to the diameter of the duct, thereby reducing the liner effectiveness. As a result, there is a need for increased impedance model fidelity (i.e., a reduction in impedance prediction uncertainty) such that optimum liner impedances can be more consistently achieved. The most significant parameter used to describe the pertinent acoustic properties of these liners is acoustic impedance, and it is the uncertainty in the measurement and prediction of this parameter that is the focus of this study. NASA Langley has recently completed an investigation of a number of impedance prediction models currently in use, with a goal of assessing the current state-of-the-art to determine an optimum approach for further enhancements, whether in experimental or predictive methods. NASA Langley is also in the process of implementing a new test facility, the Liner Technology Facility, to extend its acoustic liner measurement capabilities. Two key waveguides in this new facility will be a grazing flow impedance tube and a curved duct test rig, each of which will contain enhancements over waveguides currently in use.

In the following, a Two-Parameter (TP) model is presented for prediction of the acoustic impedance of perforateover-honeycomb acoustic liners. The two-parameter descriptor refers to the two groupings of input parameters, geometric and aeroacoustic, which control how the facesheet geometry and fluid physics contribute to the facesheet flow resistance. In total, there are four geometric and six aeroacoustic parameters that make up the two parameter groups used in the TP model. For the current study, geometric parameters are measured on a representative perforateover-honeycomb acoustic liner, while aeroacoustic parameters are determined from measurements conducted with this liner in the NASA Langley normal (NIT) and grazing (GIT) incidence tubes. The attractiveness of this model resides in the purely non-acoustic measurements required to compute the flow resistance, which is assumed equal to the facesheet acoustic resistance when the mean flow velocity through the facesheet is replaced by the RMS acoustic particle velocity in the model. This assumption is clearly an "engineering judgment," and results computed thereby should be interpreted accordingly. The complete acoustic impedance of the liner is determined via the combination of this predicted acoustic resistance with a predicted acoustic reactance based on classical acoustic resonator theory.

The aeroacoustic parameters mentioned above are also combined with complex acoustic pressures measured in the NIT and GIT to determine the measured acoustic impedance of the liner, such that the predicted and measured impedances can be directly compared. Uncertainties in the measurements of the geometric and aeroacoustic parameters are used with the TP model to compute the subsequent uncertainty of the predicted impedances. Correspondingly, uncertainties in the measurements of the aeroacoustic parameters (i.e., those used as inputs to the TP model, as well as the complex acoustic pressures) are used with the NASA Langley impedance eduction process to determine the resultant uncertainty of the measured impedances. The ranges of predicted and measured impedance uncertainty (uncertainty limits) are then compared to gain insight into the acoustic impedance prediction and measurement capabilities. In general, the predicted and measured impedances are observed to converge for frequencies at or near resonance, and to diverge for frequencies at or near anti-resonance.

Finally, a sensitivity analysis is used to further evaluate the effects of input parameter uncertainty on the corresponding predicted impedance uncertainty. For this portion of the study, the input parameter uncertainty is assumed to be equal to the measurement uncertainty observed for each of the aeroacoustic parameters during multiple repeated tests conducted in the NIT and GIT. In general, the discharge coefficient is observed to be the largest contributor to uncertainty in predicted impedance. 
In summary, the purposes of the current investigation are to (1) evaluate the TP model using uncertainty and sensitivity analyses, (2) evaluate the processes used to measure, or educe, liner impedances in the NASA Langley NIT and GIT using an uncertainty analysis, and (3) compare the results of these evaluations. Insight gained from this investigation regarding the relative importance of each input parameter will be used to guide the implementation of the new NASA Langley test capabilities, such that measurements conducted in the enhanced waveguides will be of sufficient quality to support extensions to this and other impedance prediction models.

\section{Two-Parameter Impedance Prediction Model} $\mathrm{N}$ the current investigation, a Two-Parameter (TP) model $^{1}$ is used to predict the single-layer, perforate-over-honeycomb,
liner acoustic impedance

$$
\zeta=\theta+i \chi
$$

where $\theta$ and $\chi$ are the normalized resistance and reactance components of the normalized acoustic impedance, $\zeta$. This semi-empirical model is referenced as the Two-Parameter model because of the usage of a two-parameter descriptor for the normalized acoustic resistance, which is the dominant feature of the model. The bases for development of the resistance and reactance components are provided in the following sections.

\section{A. Resistance Component, $\theta$}

The TP model assumes the thickness of the perforate facesheet is sufficiently small, relative to the shortest acoustic wavelength of interest, to be treated as a lumped element. Again, "engineering judgment" is used to determine whether the facesheet thickness is sufficiently small. For a facesheet thickness of $1 \mathrm{~mm}$ and a frequency of $10 \mathrm{kHz}$, the thickness-to-wavelength ratio is less than $3 \%$. This ratio gets progressively smaller as the frequency is decreased (wavelength is increased). Thus, the TP model is assumed to be reasonable for use with conventional perforate-overhoneycomb liners. One key implication of this assumption is that the oscillatory, or acoustic, flow through the facesheet can be treated as locally incompressible. However, this expectation may be flawed if the acoustic excitation is sufficient high and the open area ratio of the facesheet is relatively low. In addition, turbulence beneath grazing flow boundary layers can contribute greatly to the fluctuating velocity through the perforate. Thus, one basis for empirical constants used to determine the acoustic resistance in this model is the measurement of steady flows through the facesheet; i.e., the DC flow resistance. This DC flow resistance, $R_{f}$, has two components (hence, the name Two-Parameter Model), one linear and the other nonlinear, and is given as

$$
R_{f}=A+B \cdot V_{i n c}
$$

The $A$ term emphasizes the contributions of fluid viscosity and hole size to the linear resistance component, while the $B$ term accounts for contributions to the nonlinear resistance component due to vortex shedding from the edges of the holes. The flow resistance of the perforate facesheet can be determined using a conventional raylometer, in which steady flow (at velocity $V_{i n c}$ ) is passed through the material and the corresponding pressure drop, $\Delta p$, across the facesheet is measured. In this case, the flow resistance is computed using

$$
R_{f}=\Delta p / V_{i n c}
$$

It is clear that the constants $A$ and $B$ can be determined by curve fitting measured values of $R_{f}$ and $V_{i n c}$. However, it is labor intensive to make such measurements on every facesheet of interest. Instead, the TP model is based on an analytic structure inferred from the one-dimensional momentum equation, ${ }^{2}$ and is given by

$$
\theta=\frac{R}{\rho c} \equiv \frac{R_{f}}{\rho c}=\frac{a \mu t}{2 \rho c\left(\sigma C_{D}\right) d^{2}}+\frac{k_{i}+k_{e}}{2 c\left(\sigma C_{D}\right)^{2}} V_{r m s}
$$

where the incident steady flow velocity, $V_{i n c}$, is replaced with the RMS acoustic particle velocity, $V_{r m s}$, and $\mu, \rho$ and $c$ are the viscosity, density and sound speed, respectively. The empirical constants $k_{i}$ and $k_{e}$ account for entrance and exit end effects of the facesheet holes, respectively, and their sum is taken to have the value of unity. The discharge coefficient, $C_{D}$, accounts for vorticity-related losses at the hole exit, and is generally set to a value of 0.76 for sharpedged holes. In this derivation, the first term on the right can be found in Darcy's law ${ }^{3}$ for seepage flow through porous media and the second term on the right can be found in orifice metering theory. ${ }^{4}$ For laminar flow in circular orifices, $a$ is assigned ${ }^{3}$ a value of 32 . The discharge coefficient $C_{D}$ is set to a value of 0.77 to correspond to the value educed 
from DC flow resistance measurements ${ }^{5}$ for a number of liners with similar construction to that used in the current investigation.

However, the discharge coefficient is Reynolds number and $t / d$-dependent (sheet thickness-to-hole diameter ratio), and also may depend on porosity due to interference between flows through adjacent holes if the holes are in sufficiently close proximity. As shown in Eq. 4, no explicit hole interaction effects are included. The $k_{i}$ and $k_{e}$ terms are related to hole interaction effects but, by setting their sum to unity, these interactions are ignored. Instead, the TP model incorporates the open area ratio, $\sigma$, as a scaling parameter. These dependencies are also demonstrated by the measurement of the nonlinearity factor, $N L F$, which is taken to be the ratio of the flow resistance at an incident velocity of $2 \mathrm{~m} / \mathrm{s}$ to that at $0.2 \mathrm{~m} / \mathrm{s}$. For linear materials, $N L F$ takes the value of unity because the flow through the holes remains laminar to higher velocities, thereby reducing the contribution of the vorticity-related losses in the $B$ term. Correspondingly, if the $t / d$-ratio is increased, the $A$ term becomes dominant. However, facesheets commonly used in conventional aircraft are nonlinear, with $N L F$ values up to at least two.

The last effect to be included in the computation of acoustic resistance via the TP model is that due to grazing flow; i.e., the flow through the aircraft engine nacelle. In the absence of mean flow, Eq. 4 provides the full resistance component, $\theta$. On the other hand, if the liner is in the presence of mean flow in which the boundary layer displacement thickness, $\delta^{*}$, is less than the hole diameter, $d$, the grazing flow effect is computed using the classical Rice-Heidelberg model $^{6}$ given by

$$
\frac{R_{g f}}{\rho c}=\frac{M_{\text {ave }}}{\left(2+1.256 \frac{\delta^{*}}{d}\right) \sigma}
$$

where $R_{g f}$ is the grazing flow resistance and $M_{\text {ave }}$ is the free-stream, average Mach number. Clearly, this term will become dominant at high mean flow velocities. Thus, when the boundary layer displacement thickness approaches the hole diameter and the mean flow velocity becomes sufficiently large, the grazing flow effect is roughly approximated by

$$
\frac{R_{g f}}{\rho c} \simeq \frac{M_{\text {ave }}}{3 \sigma}
$$

which is a "back-of-the-envelope" design rule for conventional perforates at typical inlet and exhaust duct Mach numbers. In summary, the complete resistance component is given by

$$
\theta=\frac{a \mu t}{2 \rho c\left(\sigma C_{D}\right) d^{2}}+\frac{k_{i}+k_{e}}{2 c\left(\sigma C_{D}\right)^{2}} V_{r m s}+\frac{R_{g f}}{\rho c}
$$

where the $R_{g f} / \rho c$ term is provided by Eqs. 5 or 6 , depending on the grazing flow Mach number.

\section{B. Reactance Component, $\chi$}

For frequencies well away from resonance, the dominant contributor to the acoustic reactance of the single-layer liner is the cavity depth, $h$. For the relevant frequency range, the honeycomb cell depth (cavity depth) is modeled as a transmission line with rigid termination. The acoustic reactance component due to such a cavity is given by

$$
\chi_{c a v}=-\cot (k h)
$$

The remaining contributor to the acoustic reactance is the normalized mass reactance due to the perforate facesheet, and is taken to be

$$
\chi_{m}=\frac{k(1+\varepsilon d)}{\sigma C_{D}}
$$

where $\varepsilon d$ is the hole length end correction and

$$
\varepsilon=\frac{0.85(1-0.7 \sqrt{\sigma})}{1+305 M_{\text {ave }}^{3}}
$$

In summary, the complete acoustic reactance is given by

$$
\chi=-\cot (k h)+\frac{k(1+\varepsilon d)}{\sigma C_{D}}
$$

Thus, the total acoustic impedance, $\zeta$, is given by the combination of Eqs. 7 and 11 . 


\section{Experimental Methods}

S described above, the TP impedance prediction model contains a number of geometric and aeroacoustic input A parameters. While some of these are set according to semi-empirical models, a number of these parameters can be measured via direct or indirect means. A series of tests were conducted to determine the uncertainty in the measurements of these parameters, and these uncertainties are propagated through the TP model to determine the resultant uncertainty in the predicted impedance. Measurements of additional parameters were also made in the NASA Langley normal and grazing incidence tubes (NIT and GIT), such that the measured data (and corresponding uncertainties) can be used to determine the corresponding uncertainty in the measured impedance. This section describes the baseline liner chosen for this process, and also provides a brief discussion of the test facilities.

\section{A. Evaluation Liner}

A single-layer, conventional, perforate-over-honeycomb liner geometry (sketch provided in Fig. 1) was chosen for the current investigation. This liner geometry was chosen because it had been selected by industry ${ }^{5}$ as representative of liners commonly used in current aircraft engine nacelles. Two configurations ( 50.8 by $50.8-\mathrm{mm}$ and 50.8 by $406.4-\mathrm{mm}$ surface areas) were fabricated from the same parent material, using identical fabrication techniques, such that results measured with one sample could be assumed to be representative of those expected for the other sample. The pertinent geometric parameters used to describe this liner are:

1. Facesheet thickness $(t)-0.81 \mathrm{~mm}$

2. Hole diameter $(d)-0.97 \mathrm{~mm}$

3. Cavity depth $(h)-38.18 \mathrm{~mm}$

4. Open area ratio $(\sigma)-0.089$

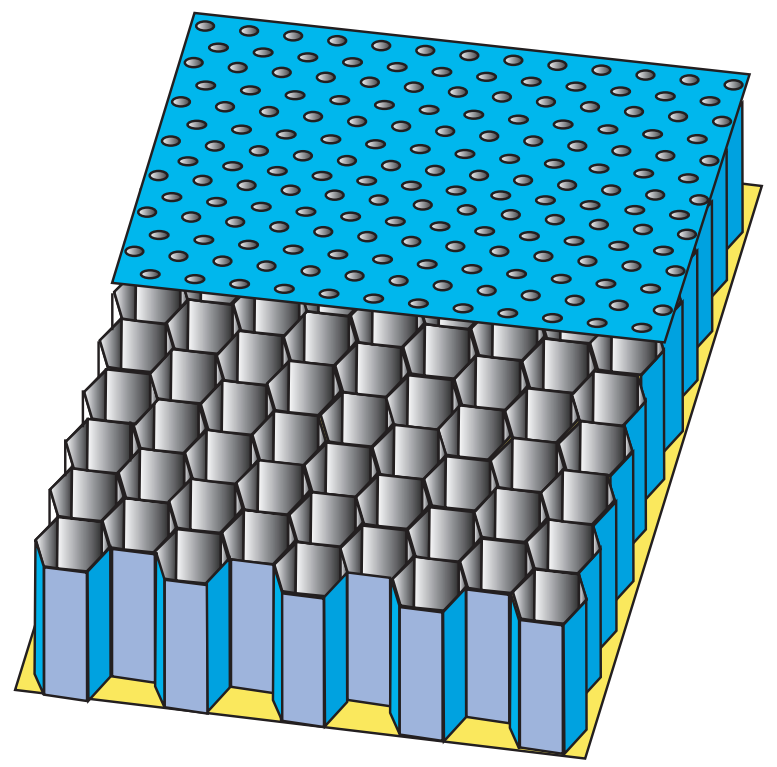

Figure 1. Sketch of perforate-over-honeycomb liner.

\section{B. Normal and Grazing Incidence Waveguides}

Initial tests were conducted with the smaller $(50.8 \times 50.8-\mathrm{mm})$ sample mounted onto the NASA Langley NIT (see Fig. 2), a $0.5 \mathrm{~m}$-long, $50.8 \times 50.8$ - $\mathrm{mm}$ waveguide. Impedances were measured in $0.1 \mathrm{kHz}$ increments (single tone at a time) from 0.4 to $3.0 \mathrm{kHz}$ using the Two-Microphone Method. ${ }^{7,8}$ At each test frequency, sound pressure levels (SPL at the reference microphone; see Fig. 2) of 120 and $140 \mathrm{~dB}$ (target SPL's) were obtained. 


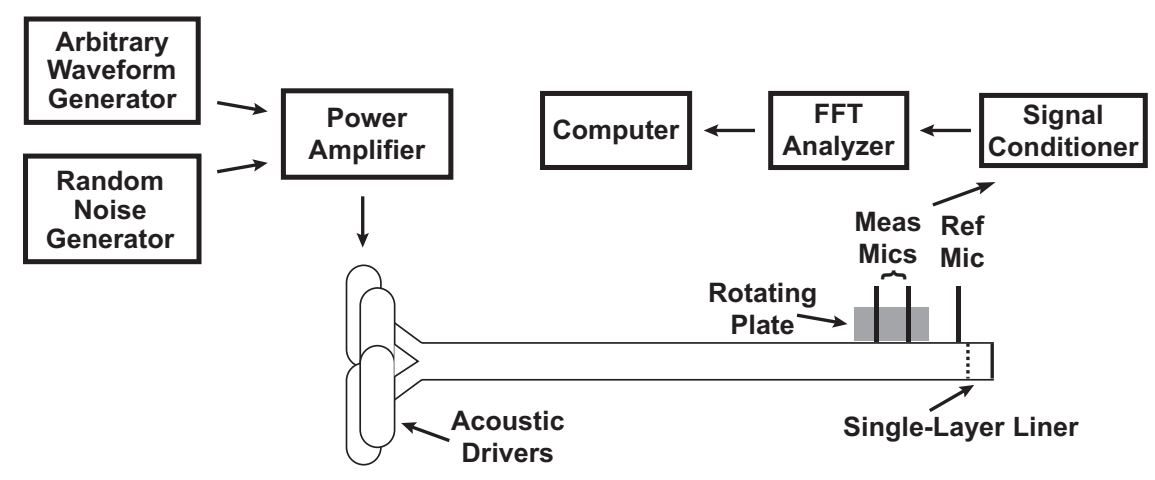

Figure 2. Sketch of Langley Normal Incidence Tube with supporting instrumentation.

Tests were also conducted in the NASA Langley GIT (see Fig. 3), a 6 m-long, 50.8 x 50.8-mm waveguide used to measure acoustic liner performance within a controlled grazing flow environment. Air flows from left to right through the test section, in which the upper wall contains the acoustic liner. Heated, pressurized air is supplied at the duct entrance via a plenum chamber, and is combined with a vacuum pump at the duct exit to allow separate control of the static pressure and Mach number. Acoustic testing is conducted at near-ambient conditions with grazing flows up to a centerline Mach number of 0.475 . For the current investigation, four $120-\mathrm{W}$ electromagnetic acoustic drivers mounted upstream of the plenum generated source $S P L$ 's (as measured by a lower-wall microphone located $101.6 \mathrm{~mm}$ upstream of the leading edge of the liner) of 120 and $140 \mathrm{~dB}$ (target $S P L$ 's, one tone at a time), at frequencies of 0.5 to $3.0 \mathrm{kHz}$, in increments of $0.5 \mathrm{kHz}$. Twenty-seven microphones mounted along the lower wall (opposite the liner) of the GIT were used to measure the resultant acoustic pressure profile (amplitude and phase), and the impedance of the sample liner was determined using NASA Langley's impedance eduction technique. ${ }^{9}$

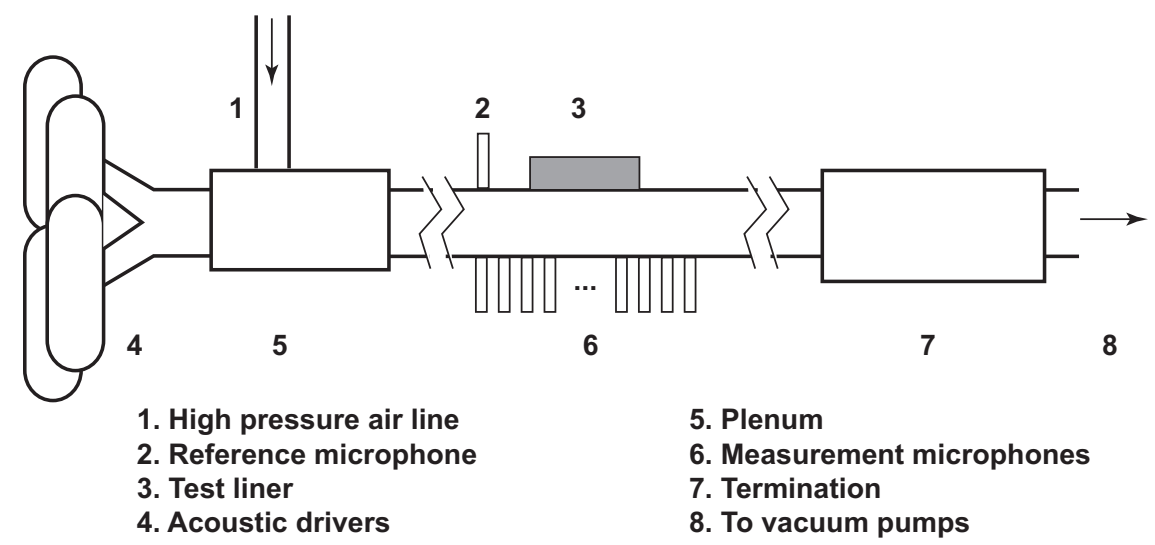

Figure 3. Sketch of Langley Grazing Incidence Tube.

Meaningful statistical metrics were calculated for data acquired in the NIT and GIT. Repeat runs were conducted at different times of the year, and the test liner of interest was always removed and reinstalled between tests. The 50.8 x 50.8-mm liner was tested 14 times in the NIT at each test condition (i.e., one frequency and one SPL), and the 50.8 $\mathrm{x}$ 406.4-mm liner was tested at least eight times in the GIT at each test condition of interest (i.e., one frequency, one source $S P L$ and one Mach number).

\section{Uncertainty Analyses}

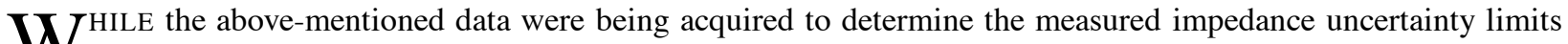
(i.e., the upper and lower bounds of the $95 \%$ confidence interval), the related aerodynamic parameters were recorded. These aerodynamic parameters were then combined with measured geometric parameters to compute the predicted impedance uncertainty limits for the selected single-layer acoustic liner, such that the predicted and measured impedance uncertainty limits could be compared. 


\section{A. Two-Parameter Impedance Prediction Model Inputs}

In what follows, a brief description is provided of how each input parameter was measured, along with a description of how the uncertainty in that parameter was determined. (Recall that the model is named for the two parameter groups, geometric and aeroacoustic, which consist of ten input parameters that can be measured.) For those parameters that were measured at least once per test (between eight and fourteen tests), the mean and standard deviation were computed in the classical manner. For any parameters that were determined to be independent of any particular test, the method chosen for estimating its mean and standard deviation is included with the description below. The resultant means and standard deviations (denoted StDev to distinguish from the open area ratio, $\sigma$ ) are provided in Table 1 for aeroacoustic parameters, and are listed with the parameter description for geometric parameters.

\section{Aeroacoustic Parameters}

These parameters are associated with the aeroacoustic environment. The following provides a description of how each parameter is "measured" in either the NIT or GIT.

1. Static pressure $\left(P_{s}\right)$ :

(a) The atmospheric pressure in the test facility is recorded prior to each test in the NIT. The static pressure for NIT tests is taken to be the atmospheric pressure measured in the test facility.

(b) A pitot-static probe mounted in the center of the GIT airway, downstream of the test window, is used to record the static pressure at the beginning of each test.

2. Static temperature $\left(T_{s}\right)$ :

(a) The static temperature for NIT tests is taken to be the room temperature in the test facility.

(b) The static temperature in the GIT is recorded with the previously mentioned pitot-static probe in the center of the GIT airway.

3. Average Mach number $\left(M_{\text {ave }}\right)$ :

(a) The Mach number in the NIT is zero.

(b) The average Mach number in the GIT is based on the measured centerline Mach number, computed using the pressure ratio measured with the same pitot-static probe (see item 1.b above). In an earlier test ${ }^{10}$ conducted in the GIT, the mean flow profile was measured across three axial planes using a motorized, traversing pitot probe and a wall mounted static pressure tube. These planes were located just upstream of the liner leading edge, near the axial midpoint of the liner section, and near the liner trailing edge. An average Mach number was computed at each axial plane by integrating over the entire plane. These three Mach numbers (one per axial plane) were then averaged to get a single Mach number $\left(M_{\text {ave }}\right)$ that is used in all successive calculations. The $M_{\text {ave }}$ 's were correlated with the corresponding centerline Mach numbers (as measured at the pitot-static probe location downstream of the test window) to get a curve fit of $M_{\text {ave }}$ as a function of centerline Mach number. Thereafter, the centerline Mach numbers were measured, and the corresponding average Mach numbers were computed based on this curve fit.

4. Boundary layer displacement thickness $\left(\delta^{*}\right)$ :

(a) The boundary layer displacement thickness in the NIT is zero.

(b) For the GIT tests, this parameter was assumed to be correlated with the centerline Mach number as determined in the earlier test. ${ }^{10}$ In a manner similar to that described above for $M_{\text {ave }}, \delta^{*}$ was computed based on the velocity profiles in each of three axial planes, and its average value was correlated with the average Mach number. During the current tests, this parameter was not "measured," but was instead "assumed" to be solely a function of the target $M_{\text {ave }}$. As such, it was assumed that the range of boundary layer displacement thickness over the length of the liner (i.e., difference between values at the upstream and downstream planes) was assumed to represent the full range of "uncertainty" for this parameter; i.e $\pm 3 \cdot \operatorname{StDev}\left(\delta^{*}\right)$.

5. Discharge coefficient $\left(C_{D}\right)$ : In a previous study, ${ }^{5}$ a number of liners were fabricated using the same manufacturing process as was used for the liner evaluated in the current investigation. DC flow resistance measurements were conducted to educe the discharge coefficient for each sample. The mean and standard deviation reported for that study [Mean $\left(C_{D}\right)$ of 0.771 and $\operatorname{StDev}\left(C_{D}\right)$ of 0.135$]$ are used in the current investigation. 
6. Excitation level $(S P L)$ :

(a) A reference microphone mounted $6.35 \mathrm{~mm}$ from the surface of the liner in the NIT is used to record the sound pressure level. This microphone is considered to be sufficiently close to the surface to provide a satisfactory measure of the excitation level at the liner surface.

(b) A microphone mounted in the lower wall of the GIT at the "computational source plane" (101.6 mm upstream of the liner leading edge) records the source $S P L$.

The $S P L$ is reset for each frequency of interest via an automated signal conditioning process (feedback loop) that monitors the output of the reference (for NIT) or source-plane (for GIT) microphone. This SPL can deviate from the target value while remaining within preset tolerance limits. For each case described in Table 1, the source $S P L$ values recorded for each frequency during each test are used to compute frequency-independent mean and standard deviations. (As a side note, the GIT computational domain used in the current investigation is of shorter axial extent than that typically reported. ${ }^{10}$ A problem in the test setup necessitated the usage of only 27 of the lower wall microphones, and also resulted in larger $S P L$ variation than that typically achieved in this test rig.)

Table 1. Aeroacoustic Parameters

\begin{tabular}{|c|c|cc|cc|cc|cc|cc|}
\hline & & \multicolumn{2}{|c|}{$P_{s}, \mathrm{~Pa}$} & \multicolumn{2}{c|}{$T_{s}, \mathrm{k}$} & \multicolumn{2}{c|}{$M_{\text {ave }}$} & \multicolumn{2}{c|}{$\delta^{*}, \mathrm{~mm}$} & \multicolumn{2}{c|}{$S P L, \mathrm{~dB}$} \\
\hline Case & Test Rig & Mean & StDev & Mean & StDev & Mean & StDev & Mean & StDev & Mean & StDev \\
\hline 1 & NIT & 101601 & 611 & 295.8 & 0.8 & 0 & - & 0 & - & 120.0 & 0.2 \\
2 & NIT & 101438 & 810 & 295.4 & 1.3 & 0 & - & 0 & - & 140.0 & 0.4 \\
3 & GIT & 101701 & 387 & 295.8 & 0.9 & 0 & - & 0 & - & 122.3 & 1.6 \\
4 & GIT & 101686 & 485 & 295.8 & 0.6 & 0 & - & 0 & - & 140.5 & 1.1 \\
5 & GIT & 101687 & 790 & 295.3 & 0.3 & 0.252 & 0.0012 & 1.75 & 0.102 & 121.3 & 0.9 \\
6 & GIT & 101933 & 862 & 295.2 & 0.2 & 0.251 & 0.0012 & 1.75 & 0.102 & 140.7 & 0.8 \\
7 & GIT & 101839 & 783 & 293.8 & 0.2 & 0.400 & 0.0007 & 1.75 & 0.102 & 120.2 & 1.6 \\
8 & GIT & 101823 & 754 & 293.8 & 0.2 & 0.400 & 0.0006 & 1.75 & 0.102 & 140.6 & 1.2 \\
\hline
\end{tabular}

\section{Geometric Parameters}

As mentioned earlier, each configuration ( $50.8 \times 50.8-\mathrm{mm}, 50.8 \times 406.4-\mathrm{mm})$ was fabricated from the same parent material. Thus, it was assumed that the geometric parameters have the same values (means and standard deviations) for either configuration. These parameters were measured on the smaller samples, for which they could more conveniently be attained. The following parameters were measured at ten randomly selected locations across the sample surface. The corresponding means and standard deviations computed from these measurements are provided with each parameter. Clearly, this process is not sufficiently detailed to distinguish between random and random and systematic variability. Thus, for the purposes of the current study, all variability is assumed to be random.

1. Facesheet thickness $(t)$ : The thickness was measured using a computer-aided system that allows the user to move a probe until it makes contact with a surface and then records the location in a 3-D coordinate system. The facesheet thickness was determined by taking the difference between the results from measurements taken in this manner from both sides of the facesheet.

$$
\operatorname{Mean}(t)=0.81 \mathrm{~mm}, \quad \operatorname{StDev}(t)=0.025 \mathrm{~mm}
$$

2. Cavity depth $(h)$ : The cavity depth was measured with the same contact probe system described above.

$$
\operatorname{Mean}(h)=38.18 \mathrm{~mm}, \quad \operatorname{StDev}(h)=0.076 \mathrm{~mm}
$$


3. Hole diameter $(d)$ : A set of precision pins was used to determine the hole diameter of ten randomly selected holes. The outer diameter of the largest pin that would fit through a hole was used as the diameter. All holes were observed to have the same diameter. The same precision pin provided the ideal fit for each of the holes included in the evaluation.

$$
\operatorname{Mean}(d)=0.97 \mathrm{~mm}, \quad \operatorname{StDev}(d)=0.000 \mathrm{~mm}
$$

4. Open area ratio $(\sigma)$ : This parameter is a function of the hole diameter and the distances between adjacent rows and columns of holes. The mean and standard deviation of the hole diameters were computed based on measurements described above. The distance between adjacent rows and columns was determined using the contact probe system described above. The open area ratio mean was then computed using

$$
\sigma=\frac{\pi d^{2}}{4 s_{r} s_{c}}
$$

where $s_{r}$ and $s_{c}$ are the row and column separation distances, respectively. The mean open area ratio is taken to be the value computed using the mean values of the corresponding parameters $\left(d, s_{r}\right.$ and $\left.s_{c}\right)$. Time limitations prohibited the acquisition of a detailed set of measurements sufficient to determine systematic variations. Instead, these parameters are assumed to be randomly distributed, and therefore uncorrelated, such that the standard deviation is given by

$$
\operatorname{StDev}(\sigma)=\left(\operatorname{Mean}^{2}(\sigma)\left[\left(\frac{2}{d}\right)^{2} \operatorname{Var}(d)+\left(\frac{1}{s_{r}}\right) \operatorname{Var}\left(s_{r}\right)+\left(\frac{1}{s_{c}}\right) \operatorname{Var}\left(s_{c}\right)\right]\right)^{0.5}
$$

where Mean, StDev and Var represent the mean, standard deviation and variance of the selected parameter.

$$
\operatorname{Mean}(\sigma)=0.089, \quad \operatorname{StDev}(\sigma)=0.002
$$

\section{B. Two-Parameter Impedance Prediction Model}

Monte-Carlo type simulations were used to obtain statistical metrics for the TP model. Thirty-one simulations were conducted for each condition of interest. This number was chosen such that the results of the simulations could be assumed to fall within \pm 2 standard deviations from the mean with $95 \%$ confidence. ${ }^{11}$ These simulations were conducted based on mean input parameters with randomness assigned commensurate with that measured in either the NIT or GIT. Thus, two conditions were evaluated for comparisons with NIT measurements - source (target) SPL's of 120 and $140 \mathrm{~dB}$. Correspondingly, six conditions (combinations of source SPL and average Mach number) were evaluated for comparisons with GIT measurements; source (target) SPL's of 120 and $140 \mathrm{~dB}$, average Mach numbers of $0.00,0.25$ and 0.40 . Computations with the prediction model were conducted at frequencies of 0.4 to $3.0 \mathrm{kHz}$, in increments of $0.1 \mathrm{kHz}$.

A randomly selected value for each aeroacoustic and geometric input parameter was used for each simulation with the impedance prediction model. Also, to mimic the reality of the measurements conducted in the NIT and GIT, a randomly selected value for the excitation level was used at each frequency within an individual simulation. The mean and standard deviation of the impedances computed for these thirty-one simulations were then used to compute $95 \%$ confidence intervals ${ }^{11}$ (abbreviated as PCI, for prediction confidence interval, in the remainder of this document) at each frequency of interest. In other words, the user can have $95 \%$ confidence that the next simulation conducted using this same approach would provide an impedance that falls somewhere within the specified interval. It should be noted that the $95 \%$ confidence interval is centered on the mean of the thirty-one simulations. To minimize clutter in the following figures, this mean spectrum is not displayed. This choice was made to emphasize the fact that it is the range of possibilities that is of utmost importance to the user desiring to predict the outcome of the next simulation.

\section{Statistical Metrics of Measured (Educed) Impedances}

As indicated earlier, multiple tests were performed at each test condition of interest. For each test, a measured impedance spectrum was educed, using either the Two-Microphone Method in the NIT or the NASA impedance eduction method in the GIT. The mean and standard deviation of the measured impedance at each frequency of interest were then determined, and the corresponding 95\% confidence interval (abbreviated as MCI, for measurement confidence interval, in the remainder of this document) on the measured data was computed. 


\section{Comparison of Predicted and Measured Impedances}

Figures 4-9 show predicted and measured impedance spectra comparisons. In each figure, the two blue curves represent the lower (solid) and upper (dashed) limits of the 95\% confidence interval (PCI) computed using the TP model. The two red curves represent the lower (solid) and upper (dashed) limits of the corresponding $95 \%$ confidence interval (MCI) for the measured data. For comparisons based on NIT measurements, a $0.1 \mathrm{kHz}$ increment is used, whereas a $0.5 \mathrm{kHz}$ increment is used for GIT comparisons. These increments are based on the frequency intervals used for the impedance measurement tests.

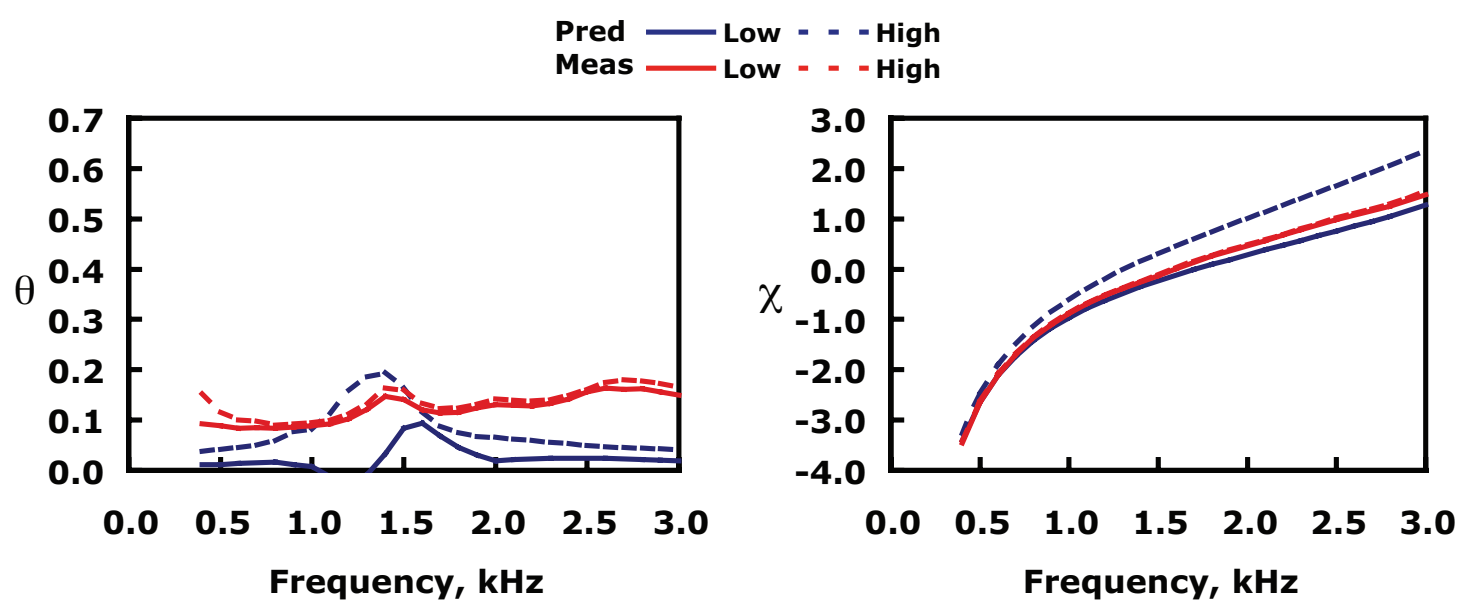

Figure 4. Comparison of $95 \%$ confidence intervals for predicted and measured impedance spectra; NIT measurements, 120 dB source $S P L$; Blue curves represent lower and upper bounds of $95 \%$ confidence intervals for predicted impedance - lower curve in (a) is clipped at zero since resistance cannot take negative value; Red curves represent lower and upper bounds of $95 \%$ confidence intervals for measured impedance.
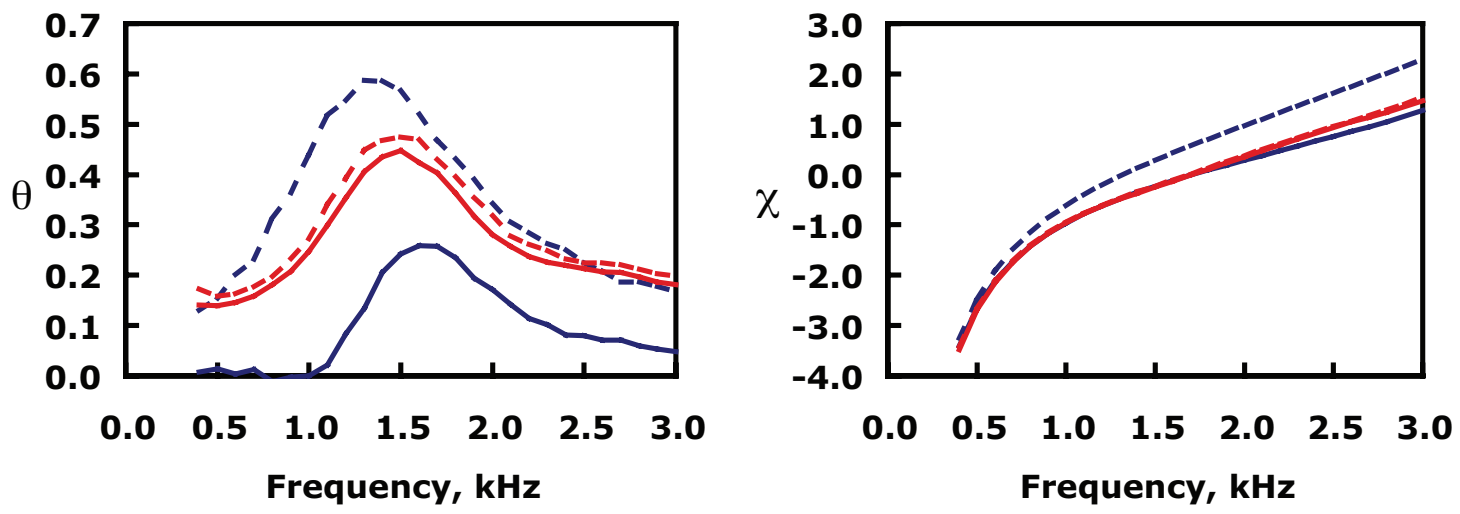

Figure 5. Comparison of $95 \%$ confidence intervals for predicted and measured impedance spectra; NIT measurements, 140 dB source $S P L$; Predicted - blue curves, Measured - red curves.

Figure 4 is based on results acquired in the normal incidence tube, with the source level set to $120 \mathrm{~dB}$. The resistance PCI is quite small (less than 0.05) at frequencies away from resonance, and grows to about 0.2 near resonance, while the reactance PCI grows from less than 0.2 at $0.4 \mathrm{kHz}$ to over 2 at $3.0 \mathrm{kHz}$. The resistance MCI is almost collapsed to a single line, and tends to be fully captured by the corresponding PCI at frequencies near resonance (where reactance has zero crossing and positive slope), but is completely above the upper limit of the PCI at frequencies away from resonance. The corresponding reactance MCI is also very small (almost collapses to single line) and is fully encompassed by the reactance PCI. When the source level is increased to $140 \mathrm{~dB}$ (Fig. 5), the resistance and reactance MCIs continue to be very small, and are almost entirely within the ranges of the corresponding PCIs. These results indicate that although the measurement uncertainty can be nearly eliminated for tests conducted in the NIT, a bias error remains between the predicted and measured results. Although this bias error is not large, it is hoped that it will 


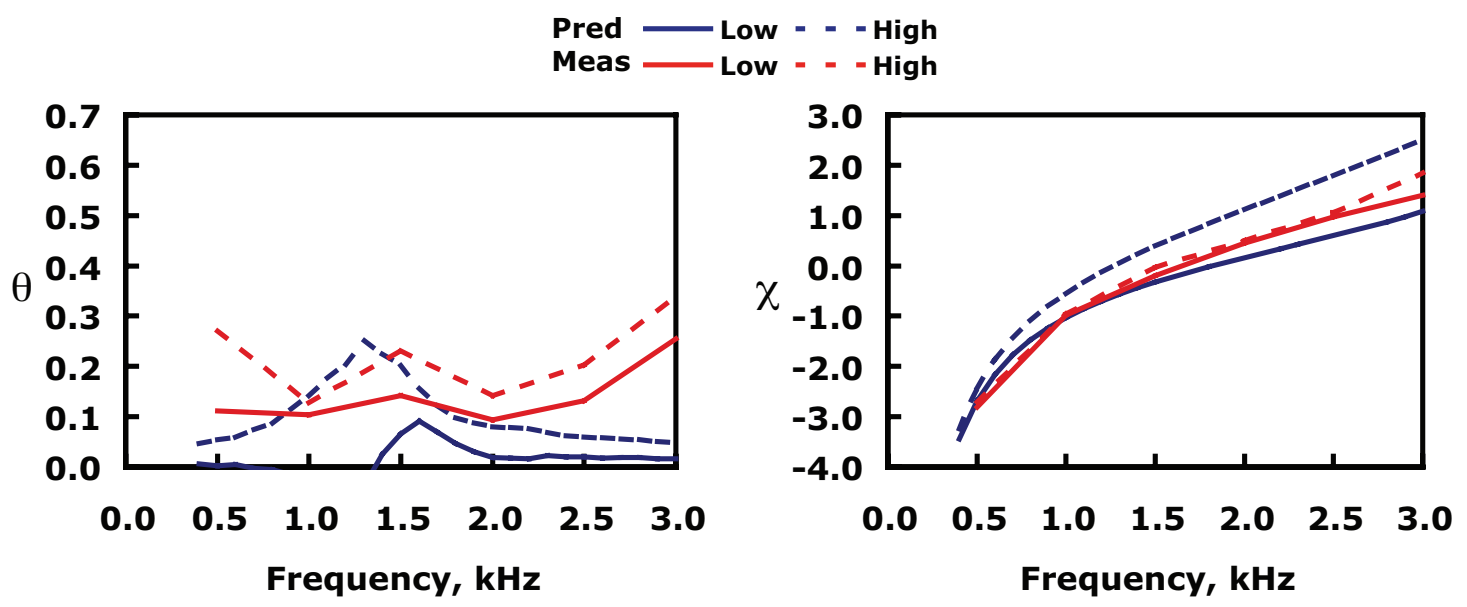

Figure 6. Comparison of $95 \%$ confidence intervals for predicted and measured impedance spectra; GIT measurements, $120 \mathrm{~dB}$ source $S P L$, no flow; Predicted - blue curves, Measured - red curves.
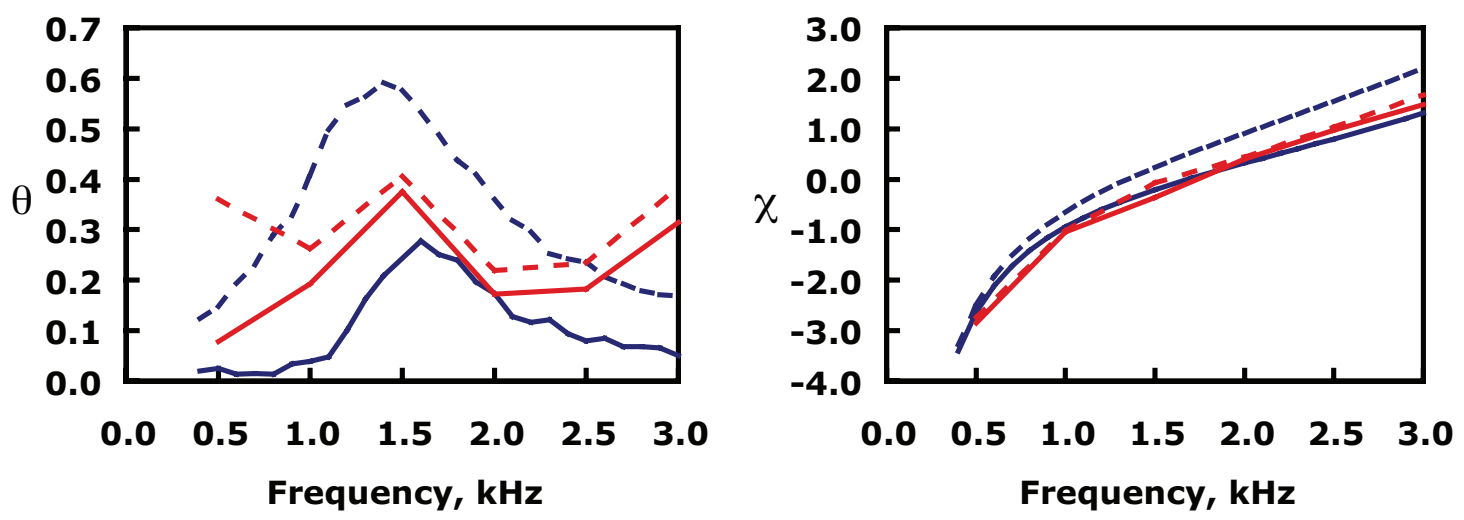

Figure 7. Comparison of $95 \%$ confidence intervals for predicted and measured impedance spectra; GIT measurements, $140 \mathrm{~dB}$ source $S P L$, no flow; Predicted - blue curves, Measured - red curves.

be reduced by future refinement of the prediction model.

Figures 6 and 7 contain results based on data acquired in the grazing incidence tube with no mean flow. As expected, the results are quite similar to those derived from NIT data. It should be noted that the impedance measurement process used with the NIT is a direct approach in which the impedance can be computed from measured data, whereas the corresponding process used with the GIT requires an iterative, finite element computation to educe the result from measured data. Thus, the slightly larger spread in the MCIs based on data acquired in the GIT is to be expected. In addition, there is a slight increase in the measured resistance at the frequency limits (500 and $3000 \mathrm{~Hz}$ ), where the frequencies approach anti-resonances. This effect is not apparent in the NIT results. Regardless, these results indicate that the TP model adequately predicts the measured impedances in the absence of mean flow.

As the mean flow velocity is increased (Mach 0.25 in Fig. 8, Mach 0.4 in Fig. 9), the predicted resistance spectra over most of the frequency range increase from approximately 0.1 at Mach 0.0 to 0.7 at Mach 0.25 , and to 1.1 at Mach 0.4. The measured resistance spectra increase even more. The resistance MCIs continue to be within the range of the corresponding PCIs at frequencies near resonance. As was observed without mean flow, the resistance MCIs are above the upper limit of the corresponding PCIs at frequencies away from resonance. While the PCIs remain relatively flat at the frequency extremes, the MCIs increase with increasing Mach number. There are a number of possible causes for this separation.

1. The TP model does not account for resistance increases that occur near anti-resonances. The limits of the frequency range used in the current investigation $(500$ and $3000 \mathrm{~Hz}$ ) each approach an anti-resonance frequency. 


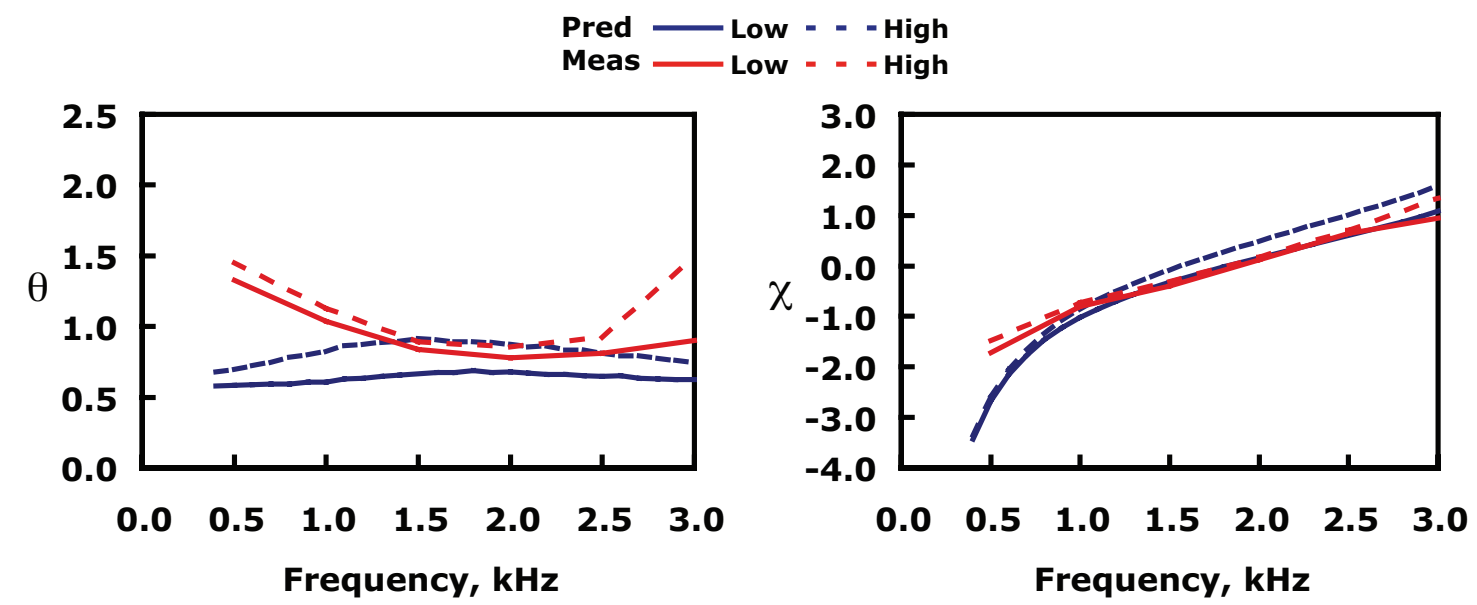

Figure 8. Comparison of $95 \%$ confidence intervals for predicted and measured impedance spectra; GIT measurements, $140 \mathrm{~dB}$ source $S P L, M_{\text {ave }}$ of 0.25 ; Predicted - blue curves, Measured - red curves.
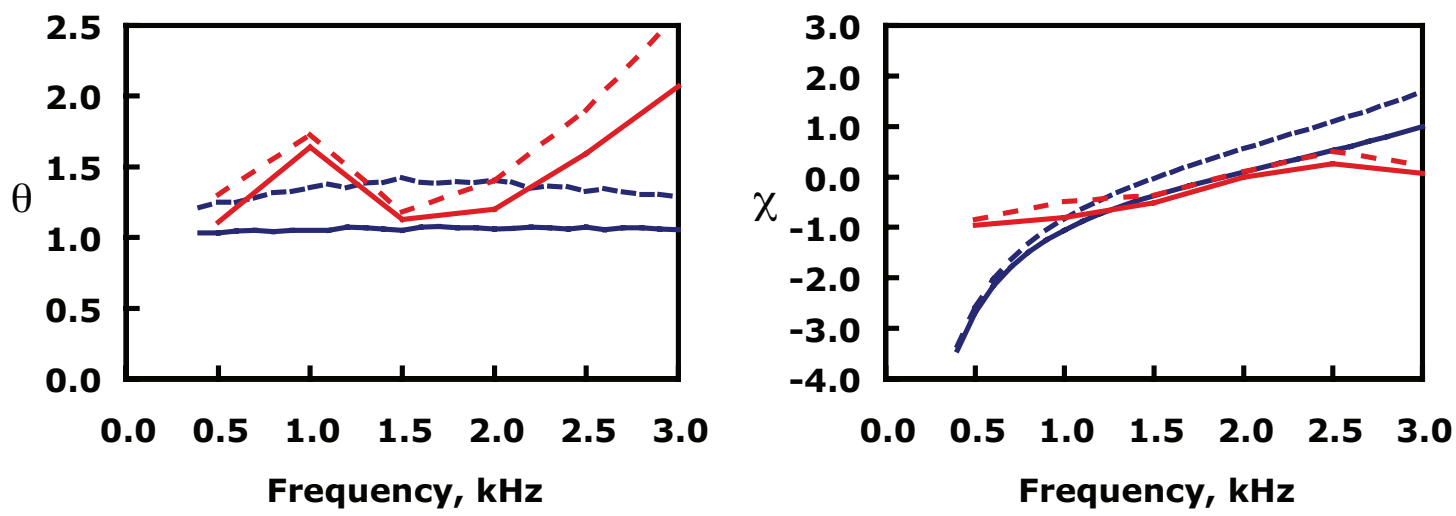

Figure 9. Comparison of $95 \%$ confidence intervals for predicted and measured impedance spectra; GIT measurements, $140 \mathrm{~dB}$ source $S P L, M_{\text {ave }}$ of 0.4; Predicted - blue curves, Measured - red curves.

2. The attenuation (difference between the $S P L$ values measured upstream and downstream of the liner) at frequencies near anti-resonance tends to be small, and is very weakly dependent on the liner impedance. In other words, there are multiple impedances that produce a similar, small attenuation. As a result, it is quite difficult for the impedance eduction method ${ }^{9}$ to "find" a unique impedance. A detailed investigation into this issue is discussed in a companion paper. ${ }^{14}$

3. At Mach 0.25 , the upper frequency limit $(3000 \mathrm{~Hz})$ is just below cut-on of a higher-order mode in the hardwall section of the duct. At Mach 0.4, a higher-order mode is cut on at this frequency. The impedance measurement process used in this investigation assumes only plane waves are present in the hardwall sections of the duct.

4. As the Mach number is increased, the effects of shear flow are expected to become increasingly important. These effects are approximated in the TP model (Eq. 5), but the impedance measurement process assumes uniform flow.

In general, these uncertainty analysis results seem reasonable. Recall that the TP model is a semi-empirical model. As liners have traditionally been designed to maximize attenuation near resonance, most predictive models have been "tuned" to this frequency range. As a result, measured and predicted values are expected to compare favorably near resonance. 


\section{Sensitivity Analysis}

T is clear from the comparisons in Figs. 4-9 that the differences between predicted and measured impedance specItra vary significantly with test condition. Further investigations into the causes of uncertainty for the impedance measurement process are provided in companion papers. ${ }^{12-14}$ In an attempt to gain insight into the bases of these disparities, a sensitivity analysis was conducted to determine the effects of varying the respective TP model input parameters over the ranges of their respective Gaussian distributions. As described earlier, there are ten input parameters $\left(P_{s}, T_{s}, M_{a v e}, \delta^{*}, t, d, \sigma, C_{D}, h, S P L\right)$ used in this model. Each input parameter is assumed to vary \pm 2 standard deviations from their respective mean values, such that the input parameter variability is based on actual measurement uncertainty observed in the current tests. The procedure for this analysis is as follows:

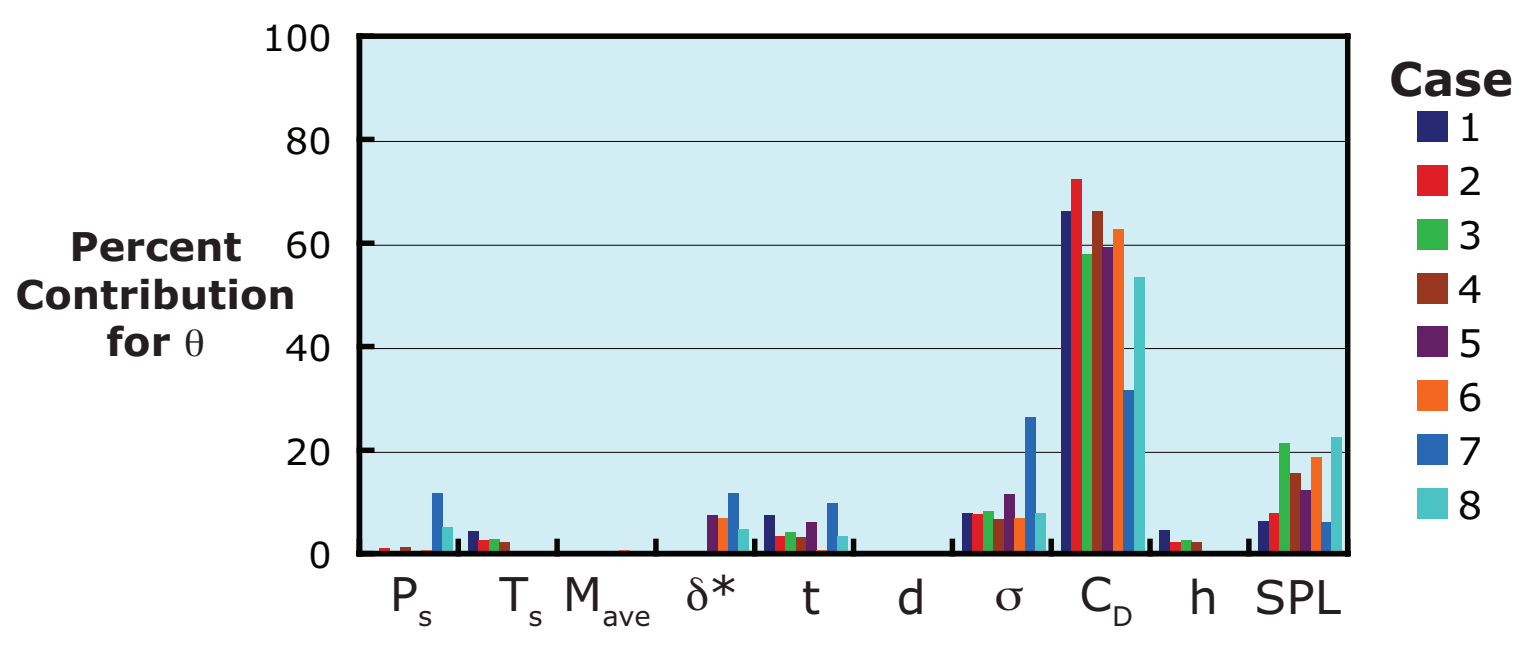

(a) Two-Parameter Model Input Parameters

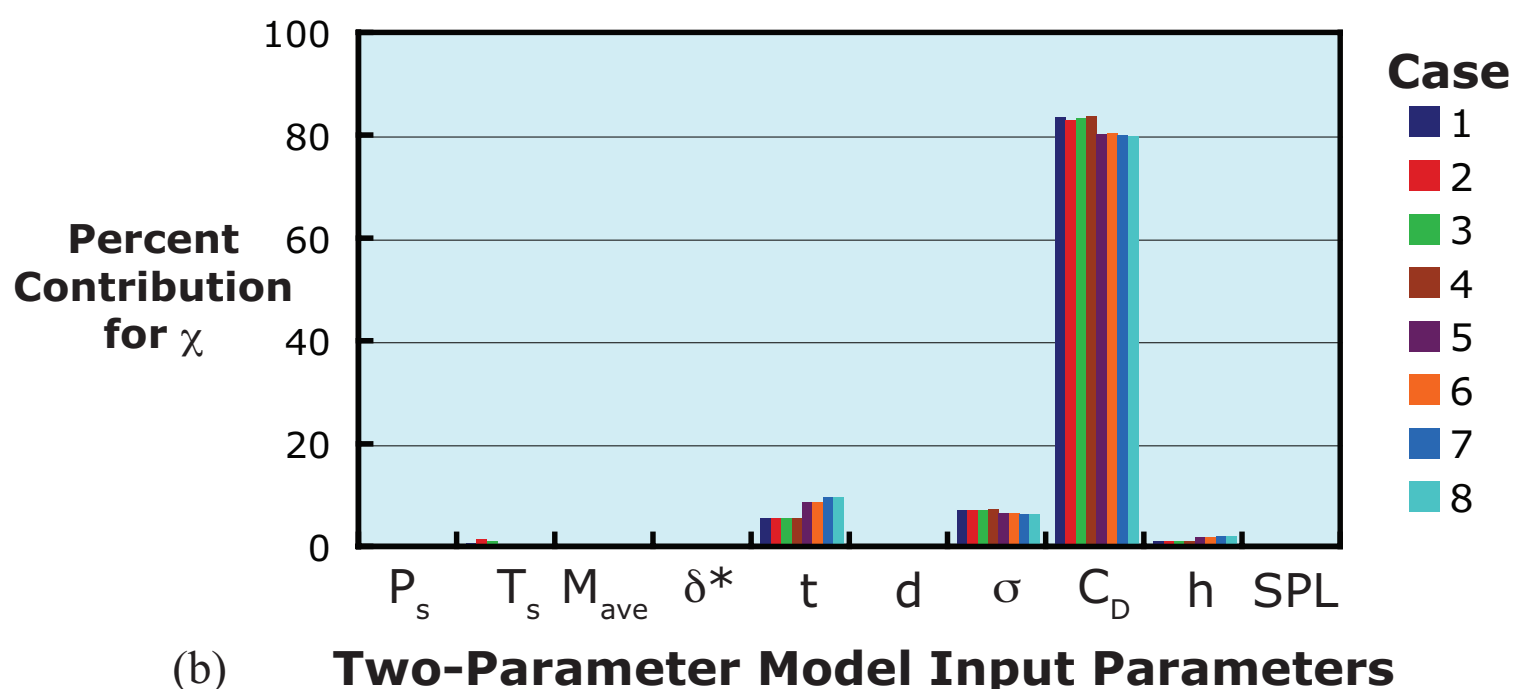

Figure 10. Percentage contributions of each input parameter to variability in impedance predicted by Two-Parameter Model, based on varying each input parameter by \pm 2 standard deviations about their respective mean values.

1. The TP model is used to compute the predicted impedance spectrum over the frequency range of 0.4 to $3.0 \mathrm{kHz}$, with each parameter set to its mean value. This step is repeated for each of the eight test cases listed in Table 1, and the predicted impedances are labeled $\zeta^{1}(f)$ through $\xi^{8}(f)$ for future reference. 
2. For each of the eight cases, the TP model is used to compute the predicted impedances with nine parameters set to their respective mean values and the remaining parameter (parameter being investigated) successively set to its minimum (mean minus 2 standard deviations) and maximum (mean plus 2 standard deviations) values. This results in two impedance spectra per input parameter for each test case, for a total of 160 impedance spectra. These impedances are labeled as $\xi_{i, j}^{L}(f)$ and $\xi_{i, j}^{H}(f)$, where the ' $i$ ' subscript indicates the case number (1 to 8), the ' $j$ ' subscript indicates the parameter number (1 to 10 ), and the ' $L$ ' or ' $H$ ' superscript indicates the low (mean minus 2 standard deviations) or high (mean plus 2 standard deviations) option, respectively.

3. The difference between the predicted impedances for the two options is computed at each frequency, $f$, using

$$
\Delta_{i, j}(f)=\xi_{i, j}^{L}(f)-\xi_{i, j}^{H}(f) \quad i=1 \text { to } 8 ; j=1 \text { to } 10
$$

4. Standard deviations are computed for the real and imaginary components of $\Delta_{i, j}(f)$, and are labeled as $S_{\theta, i, j}$ and $S_{\chi, i, j}$, respectively. These standard deviations are computed using the $\Delta_{i, j}$ values at each frequency, thereby reducing to a single set of values $\left(S_{\theta, i, j}, S_{\chi, i, j}\right)$ to represent the entire spectra. The magnitudes of $S_{\theta, i, j}$ and $S_{\chi, i, j}$ provide an indication of the relative effects of parameter ' $j$ ' on the predicted resistance and reactance, respectively, for the conditions of case ' $i$ '.

5. The values of $S_{\theta, i, j}$ and $S_{\chi, i, j}$ are compared to determine the relative sensitivity of the predicted impedance to variability in each of the ten input parameters. The relative sensitivities of the predicted resistance $\left(R_{\theta, i, j}\right)$ and reactance $\left(R_{\chi, i, j}\right)$ components are expressed as percentages of the sum of corresponding components using

$$
R_{\theta, i, j}=100 * \frac{S_{\theta, i, j}}{\sum_{j=1}^{10} S_{\theta, i, j}}, \quad R_{\chi, i, j}=100 * \frac{S_{\chi, i, j}}{\sum_{j=1}^{10} S_{\chi, i, j}}
$$

and are described as percent contributions for the resistance, $\theta$, and reactance, $\chi$, components, respectively.
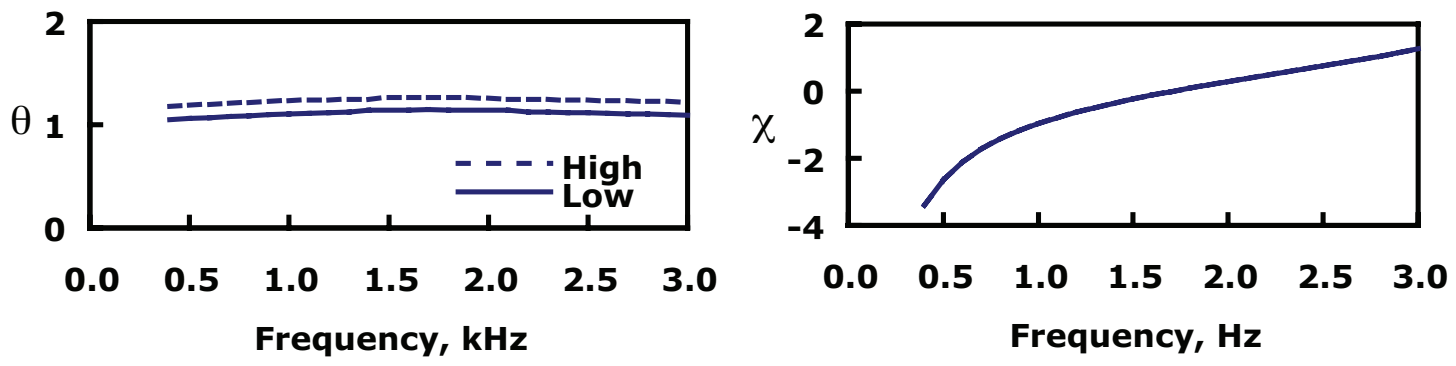

Figure 11. Contributions of boundary layer displacement thickness $\left(\delta^{*}\right)$ variability to variability of computed impedance for Case 8 - GIT test rig, $140 \mathrm{~dB}$ source $S P L, M_{\text {ave }}$ of 0.4 ; Solid and dashed curves represent lower and upper limits of predicted impedance variability due to variability of boundary layer displacement thickness by \pm 2 standard deviations about its mean.

The results of this analysis for each of the eight cases listed in Table 1 are shown in Fig. 10. Although there are certainly differences between the results for each case, some general trends emerge. It is apparent that the variability in the discharge coefficient $\left(C_{D}\right)$ was the dominant contributor to variability in the predicted resistance and reactance for the test results presented earlier in this paper. The source level $(S P L)$ variability was a significant contributor (up to about $20 \%$ ) to variability in the predicted resistance. Finally, the measured variability in open area ratio ( $\sigma$ ), facesheet thickness $(t)$ and boundary layer displacement thickness $\left(\delta^{*}\right)$ were also contributors to the variability in the predicted impedance. Based on these results, these parameters are believed to be the key contributors to the sizes of the $95 \%$ confidence intervals (PCIs) described in the "Uncertainty Analysis" section above. Of course, it should be noted that these relative effects are expected to be dependent on the selected mean values; i.e., these results are specific to this class of liners. Figure 11 is included to demonstrate how the results from Fig. 10 can be used. This figure (Fig. 11) depicts the effects of varying the boundary layer displacement thickness $\left(\delta^{*}\right)$ by \pm 2 standard deviations about its mean value, as measured in the GIT test rig with a $140 \mathrm{~dB}$ source and a Mach 0.4 mean flow (Case 8 in Table 1). As mentioned above, this amount of variability in $\delta^{*}$ causes the predicted resistance to vary by approximately 0.1 over almost the entire frequency range. There is no discernable variability (solid and dashed curves overlap) in the predicted reactance. 
These results offer guidance toward the design of future experiments. Clearly, a concerted effort should be made to minimize the measurement uncertainty related to those parameters that are the largest contributors to variability in the predicted impedance.

\section{Concluding Remarks}

This paper has presented the results of uncertainty and sensitivity analyses based on the Two-Parameter impedance prediction model. An uncertainty analysis based on Monte-Carlo-type simulations was used to evaluate the effects of simultaneous variability in each of the input parameters, while a sensitivity analysis was used to study the effects of variability in individual input parameters. Together, these analyses demonstrate the relative contributions of the respective input parameters to the predicted impedance. For the current study, values for these input parameters were based on a number of measurements conducted with a representative perforate-over-honeycomb acoustic liner in the NASA Langley normal incidence and grazing incidence impedance tubes. These measurements were also used with the NASA Langley impedance eduction process to determine the measured impedance.

Uncertainties in the measurements were propagated through the Two-Parameter model and the impedance eduction processes to compute subsequent uncertainties in the predicted and measured impedance of this liner, respectively. In general, the predicted impedance uncertainty limits (95\% confidence interval) were observed to fall within the measured impedance uncertainty limits (95\% confidence interval) for frequencies near resonance. However, while the measured reactance uncertainty limits tend to fall within the predicted reactance uncertainty limits for all but the highest Mach number, the measured resistance uncertainty limits tend to deviate from the corresponding predicted resistance uncertainty limits at frequencies away from resonance.

A sensitivity analysis was also used to provide insight into the effects of input parameter uncertainty on the corresponding predicted impedances. In general, significant variability in the discharge coefficient $\left(C_{D}\right)$ was observed to be the largest contributor to variability in the predicted impedance. This result suggests that uncertainty in the discharge coefficient was likely the largest contributor to the range of the $95 \%$ confidence intervals of the predicted impedance spectra observed in the uncertainty analysis.

Overall, these results provide insight toward proper design of future experiments. Clearly, additional effort should be made to limit the uncertainty of those parameters that contribute the most to the variability in the predicted impedance. These results will be used to guide the usage of new test rigs currently being brought on-line.

\section{References} 1991.

1“Aeroacoustics of Flight Vehicles: Theory and Practice - Volume 2: Noise Control,” NASA RP-1258, Edited by H. H. Hubbard, August,

${ }^{2}$ Wilcox, D.C.: "Basic Fluid Mechanics,” DCW Industries, April, 1997.

${ }^{3}$ Green, L., Jr. and Duwez, P.: "Fluid Flow Through Porous Materials," Journal of Applied Mechanics, March, 1951, pp. 39-45.

${ }^{4}$ Melling, T.H.: "The Acoustic Impedance of Perforates at Medium and High Sound Pressure Levels," Journal of Sound and Vibration, Vol. 29(1), 1973, pp. 1-65.

${ }^{5}$ Syed, A.A., Yu, J., Kwan, H.W. and Chien, E.: "The Steady Flow Resistance of Perforated Sheet Materials in High Speed Grazing Flows," NASA CR-2002-211749, July, 2002.

${ }^{6}$ Heidelberg, L.J., Rice, E.J. and Homyak, L.: "Experimental Evaluation of a Spinning-Mode Acoustic-Treatment Design Concept for Aircraft Inlets," NASA TP-1613, April, 1980.

${ }^{7}$ Chung, J.Y. and Blaser, D.A.: "Transfer function method of measuring in-duct acoustic properties: I. Theory," Journal of Acoustical Society of America, Vol. 68, 1980, pp. 907-921.

${ }^{8}$ Jones, M.G. and Parrott, T.L.: "Evaluation of a multi-point method for determining acoustic impedance," Journal of Mechanical Systems and Signal Processing, Vol. 3, No. 1, 1989, pp. 15-35.

${ }^{9}$ Watson, W., Jones, M., Tanner, S. and Parrott, T.: "Validation of a Numerical Method for Extracting Liner Impedance," AIAA Journal, Vol. 34(3), March, 1996, pp. 548-554.

${ }^{10}$ Jones, M.G., Watson, W.R. and Parrott, T.L.: "Benchmark Data for Evaluation of Aeroacoustic Propagation Codes with Grazing Flow," AIAA-2005-2853, May, 2005.

${ }^{11}$ Coleman, H.W. and Steele, W.G.: "Experimentation and Uncertainty Analysis for Engineers, 2nd Edition," John Wiley \& Sons, Inc., 1999.

${ }^{12}$ Watson, W.R., Nark, D.M., Jones, M.G. and Parrott, T.L.: "Assessment of 3-D Codes for Predicting Liner Attenuation in Flow Ducts," AIAA-2008-2828, May, 2008.

${ }^{13}$ Nark, D.M., Watson, W.R. and Jones, M.G.: "Uncertainty and Sensitvity Analyses of Duct Propagation Models," AIAA-2008-2832, May, 2008.

${ }^{14}$ Watson, W.R., Jones, M.G. and Parrott, T.L.: "Investigation of an Anomaly Observed in Impedance Eduction Techniques" AIAA-2008-3013, May, 2008. 